\title{
Distrofia corneana policromática posterior
}

\section{Polychromatic posterior corneal dystrophy}

Patrick Frensel de Moraes Tzelikis', Ulisses Roberto dos Santos ${ }^{2}$, Marco Antônio Guarino Tanure', Fernando Cançado Trindade ${ }^{4}$

\section{ResUmo}

Os autores descrevem quatro raros casos de distrofia corneana policromática posterior, ainda não descrito na literatura nacional. Observam-se opacidades puntiformes, policromáticas, de tamanho uniforme, localização estromal profunda, distribuídas de limbo a limbo e que não interferem na acuidade visual. É apresentada uma revisão dos casos de distrofia pré-Descemet existentes na literatura.

Descritores: Distrofias hereditárias da córnea; Córnea/patologia; Transtornos cromossômicos; Relatos de casos [Tipo de publicação]

\footnotetext{
${ }^{1}$ Assistente do Serviço de Córnea e Catarata do Hospital Oftalmológico de Brasília - HOB; Pós-graduando pela Faculdade de Medicina da Universidade Federal de Minas Gerais - UFMG - Belo Horizonte (MG) - Brasil;

${ }^{2}$ Assistente do Serviço de Córnea e Catarata do Hospital São Geraldo - Medicina da Universidade Federal de Minas Gerais - UFMG Belo Horizonte (MG) - Brasil;

${ }^{3}$ Assistente do Serviço de Córnea e Catarata do Hospital São Geraldo - UFMG; Doutor em Oftalmologia pela Faculdade de Medicina da Universidade Federal de Minas Gerais - UFMG - Belo Horizonte (MG) - Brasil;

${ }^{4}$ Professor adjunto e Chefe do Serviço de Córnea e Catarata do Hospital São Geraldo - UFMG - Belo Horizonte (MG) - Brasil; Doutor em Oftalmologia pela Faculdade de Medicina da Universidade Federal de Minas Gerais - UFMG - Belo Horizonte (MG) - Brasil.

Trabalho realizado no Hospital São Geraldo da Universidade Federal de Minas Gerais - UFMG - Belo Horizonte (MG), Brasil.
}

Recebido para publicação em: 22/05/07 - Aceito para publicação em 27/08/07 


\section{INTRODUÇÃO}

A s distrofias corneanas são alterações primárias, bilaterais, simétricas que parecem apresentar pouca ou nenhuma relação com processo inflamatório prévio ou fatores sistêmicos. Apresentam geralmente padrão hereditário autossômico dominante. ${ }^{(1)}$

As distrofias estão geralmente presentes na primeira década de vida, mas podem aparecer tardiamente. Tendem a progredir lentamente. Podem ser classificados de acordo com padrões genéticos, histopatológicos, bioquímicos, e de localização anatômica, sendo esta última a mais utilizada, identificando-as de acordo com a camada corneana envolvida. ${ }^{(2)}$

A distrofia corneana policromática posterior, devido as suas características clínicas e biomicroscópicas, é considerada um tipo de distrofia corneana préDescemet. Apresenta acometimento simétrico, bilateral, confinado a região de estroma profundo, sendo que na grande maioria dos casos não causa redução da acuidade visual. Geralmente, o paciente não possui queixas relacionadas à distrofia.

O objetivo deste trabalho é descrever quatro casos de distrofia corneana policromática posterior, uma rara distrofia de localização pré-Descemet, que apresenta poucos relatos na literatura mundial e nenhum na literatura nacional.

\section{Relato de Caso}

Caso1:Paciente J.S.G, 24 anos, sexo feminino, solteira, feodérmica, gerente administrativa, natural e residente em Belo Horizonte - MG. Procurou o Hospital São Geraldo-Universidade Federal de Minas Gerais para consulta oftalmológica de rotina, devido à pequena ametropia bilateral. Na história pregressa negava doenças oculares prévias. Não apresentava alterações sistêmicas. Seus pais, não consangüíneos, e seus dois irmãos, segundo ela, não apresentavam alterações oculares.

Ao exame oftalmológico a acuidade visual não corrigida era de 20/60 no olho direito (OD) e 20/50 no olho esquerdo (OE), sendo de 20/20 em ambos os olhos (AO) com a seguinte refração, sob cicloplegia: OD: -0,75 $=-0,25 \times 30^{\circ}$ e OE: $-0,50=-0,50 \times 180^{\circ}$.

No exame biomicroscópico observou-se em AO córnea com opacidades policromáticas, puntiformes, localizadas no estroma posterior, próximas ao plano da membrana de Descemet, de coloração variada, dimensões aparentemente uniformes e distribuídas de limbo a limbo. Entre as opacidades o estroma corneano manti-

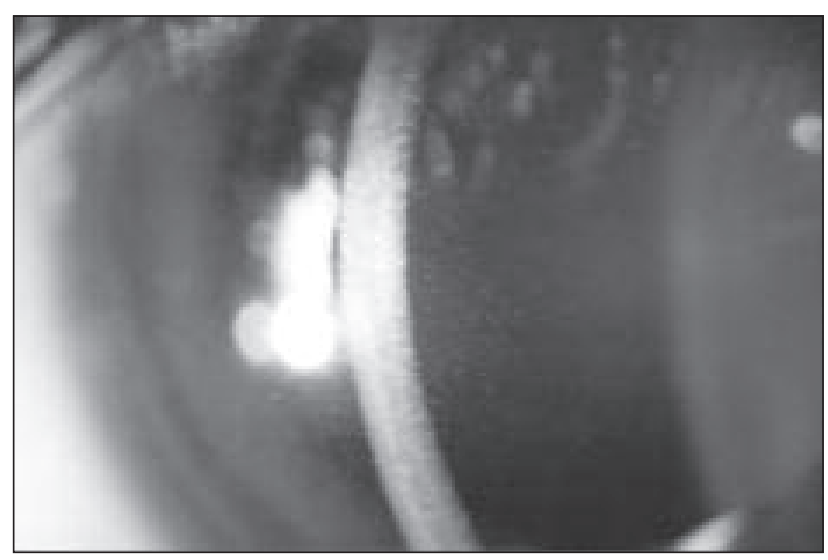

Figura 1: Caso 1 - Biomicros copia do olho direito evidenciando opacidades policromáticas, puntiformes, localizadas no estroma posterior e dimensões aparentemente uniformes

nha-se com transparência normal (figura 1).

Foi realizado microscopia especular de não contato com aparelho Iconan ${ }^{\circledR}$ (modelo Konan Noncon SP 9000). Evidenciou população endotelial no OD de 3333 cél $/ \mathrm{mm}^{2}$ e no OE de 3021 cél $/ \mathrm{mm}^{2}$, normal para a idade. A análise morfológica do endotélio evidenciou uma área celular média normal de $300 \mu \mathrm{m}^{2}$ no OD e $331 \mu \mathrm{m}^{2}$ no OE, com ausência de pleomorfismo ou polimegatismo. O que chamou a atenção foi a presença de numerosos pontos brancacentos na imagem obtida pela microscopia especular provenientes das opacidades policromáticas puntiformes localizadas na região pré-Descemet. A paquimetria óptica, medida com o mesmo aparelho Iconan $^{\circledast}$, apresentava a espessura corneana central de 600 micra no OD e 616 micra no OE (figuras 2 e 3 ).

Foi realizado exame de biomicroscopia ultrasônica (BUS) em AO que revelou discreto granular presente na região pré-Descemet, porém de aspecto praticamente normal. A motilidade ocular extrínseca, reflexos fotomotores direto e consensual, e a fundoscopia eram normais em AO.A pressão intra-ocular era de $12 \mathrm{mmHg}$ em AO às 10:00.

Seus pais e dois irmãos foram examinados. Seu pai, 50 anos, e seu irmão mais novo, 19 anos, apresentavam exatamente as mesmas alterações corneanas descritas acima, porém com as opacidades corneanas policromáticas posteriores em menor número. $\mathrm{O}$ restante do exame se mostrou todo normal. Sua mãe e seu irmão de 20 anos de idade apresentavam exame oftalmológico normal. Seus avôs não foram examinados, pois já haviam falecido.

Caso 2: Paciente M.C.S, 23 anos, sexo feminino, solteira, feodérmica, cabeleireira, natural e residente em Betim - MG. Procurou o Hospital São Geraldo - Univer- 


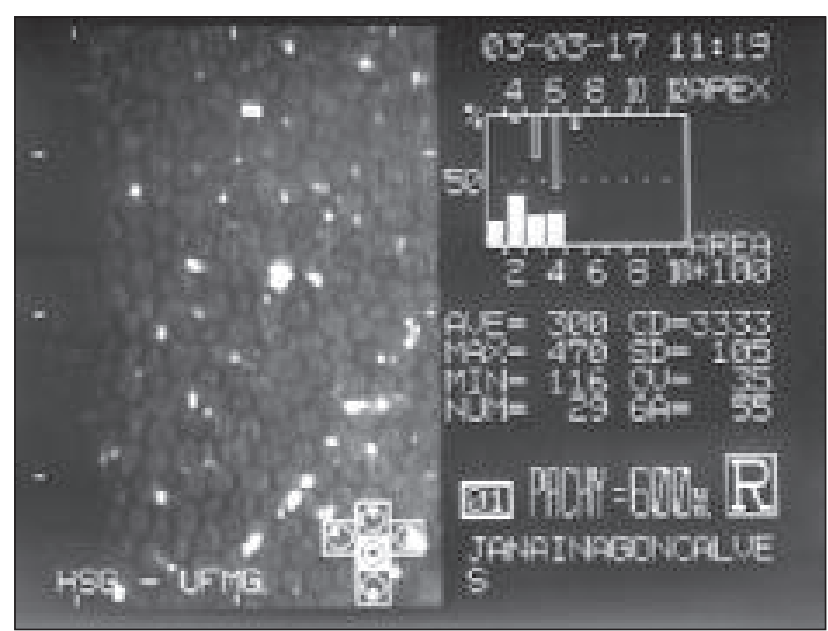

Figura 2: Caso 1 - Microscopia especular do olho direito evidenciando contagem endotelial de 3333 cél $/ \mathrm{mm}^{2}$ e presença de pontos brancacentos, distribuíd os pelo endotélio originados da op acidade policromática puntiforme pré-Descemet

sidade Federal de Minas Gerais para consulta oftalmológica de rotina, também devido à pequena ametropia bilateral. Sua história pregressa e familiar era negativa para doenças oculares. Negava cosangüinidade entre os pais. Nenhum outro membro da família foi examinado devido à dificuldade de acesso ao hospital.

Ao exame oftalmológico, a acuidade visual não corrigida era de 20/25 (0,8) em ambos os olhos (AO), sendo de 20/20 (1,0) em AO, com a seguinte refração sob cicloplegia: olho direito (OD): $+0,25=-0,50 \times 105^{\circ} \mathrm{e}$ olho esquerdo (OE): $+0,50=-0,50 \times 75^{\circ}$.

No exame biomicroscópico observou-se as mesmas alterações descritas no caso 1 em AO, com córnea apresentando opacidades policromáticas, puntiformes, localizadas no estroma posterior, próximas ao plano da membrana de Descemet, de coloração variada, dimensões aparentemente uniformes e distribuídas de limbo a limbo. Entre as opacidades, o estroma corneano mantinha-se com transparência normal (figuras 4 e 5).

A ceratometria, medida com ceratômetro Baush\&Lomb, revelou miras regulares em AO, com curvaturas nos $3,0 \mathrm{~mm}$ centrais de $41,75\left(85^{\circ}\right)$ e $41,25\left(175^{\circ}\right)$ no OD, e 42,00 $\left(80^{\circ}\right)$ e $41,50\left(170^{\circ}\right)$ no OE. A paquimetria ultra-sônica, medida com o paquímetro $\mathrm{DGH}^{\circledR}, 4000-\beta$, a espessura central corneana era de 534 micra no OD e de 531 micra no OE. A microscopia especular de contato foi feita com aparelho Tomey ${ }^{\oplus} \mathrm{EM}-1100$, versão 1.50 (Tomey AG Erlangen ${ }^{\circ}$, 1993). Evidenciou população endotelial no OD de 2262 cél $/ \mathrm{mm}^{(2)}$ e no OE de 2234 cél $/ \mathrm{mm}^{2}$ pouco abaixo do normal para a idade. A análise da morfologia do endotélio constatou uma área celular média de $442 \mu \mathrm{m}^{2}$ no OD e de $447 \mu \mathrm{m}^{2}$ no OE, com mode-

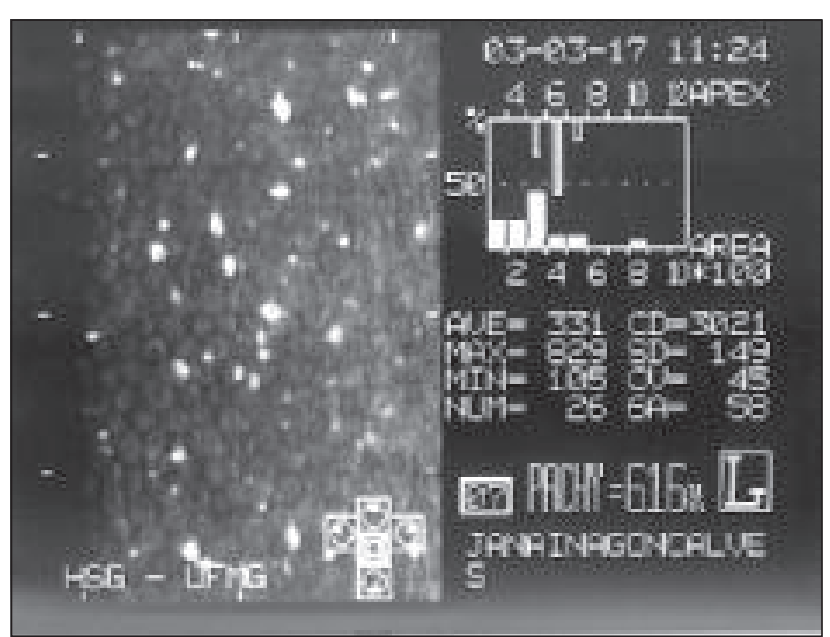

Figura 3: Caso 1 - Microscopia especular do olho es querdo evidenciando contagem endotelial de $3021 \mathrm{c} e ́ 1 / \mathrm{mm}^{2}$ e presença de pontos brancacen tos, distribuídos pelo endotélio originad os da opacidade policromática puntiforme pré-Descemet

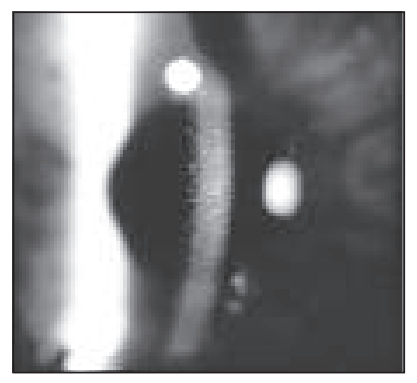

Figura 4: Caso 2 - Biomicroscopia do olho direito evidenciando opacidades policromát icas, puntiformes, localizadas no estroma posterior e dimensões ap arentemente un iformes

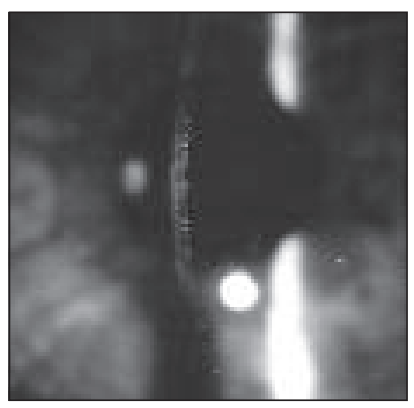

Figura 5: Caso 2 - Biomicroscopia do olho es querdo, evidenciando opacidades policromáticas, punt iformes, localizadas no estroma posterior e dimensões aparentemente uniformes

rado pleomorfismo e polimegatismo.

A motilidade ocular extrínseca, reflexos fotomotores direto e consensual, e a fundoscopia eram normais em AO. A pressão intra-ocular do OD era de 18 mmHg às 09:20 e do $\mathrm{OE}$ de $17 \mathrm{mmHg}$.

\section{Discussão}

O primeiro autor a descrever as distrofias préDescemet foi Vogt, em $1923^{(3)}$, denominando-as de distrofia farinata. A partir de então, várias formas de opacidades pré-Descemet foram sendo descritas na litera- 
tura. Em 1947, Maeder e Danis relataram um caso de distrofia pré-Descemet com opacificações de aspecto filiforme em um paciente com ceratocone ${ }^{(4)}$. Posteriormente, casos semelhantes foram observados em pacientes sem ceratocone ${ }^{(5)}$.Alterações corneanas similares foram relatadas em pacientes com distrofia polimórfica posterior $^{(6)}$ e com ictiose congênita ${ }^{(7)}$. Em 1964, Collier diagnosticou a distrofia pré-Descemet em membros de uma mesma família, sugerindo pela primeira vez uma natureza hereditária nesses $\operatorname{casos}^{(8)}$.

Grayson e Wilbrandt, em $1967^{(9)}$, descreveram 22 pacientes com distrofia pré-Descemet, observando uma tendência familiar, e propondo uma classificação baseada na forma das opacidades em três tipos: 1-córnea farinata; 2- opacidades mais largas e pleomórficas (circular, dendrítica, filiforme, linear, bumerangue) que as da córnea farinata, ocorrendo de vários padrões: anular, axial, difusa; 3- opacificações associadas com outras patologias corneanas, como ceratocone e distrofia polimórfica posterior, ou doenças dermatológicas, como ictiose.

A natureza e a patogênese das opacidades nesta distrofia permanecem obscuras. Apenas dois trabalhos até então apresentam estudo imunohistoquímico da córnea acometida ${ }^{(10-11)}$. Em 1974, Curran et al. ${ }^{(10)}$ observaram envolvimento seletivo dos ceratócitos na região do estroma profundo, sem alterações no colágeno do estroma, membrana de Descemet e endotélio corneano. Verificaram ainda a presença de um complexo lipoprotéico, semelhante à lipofucsina, comumente encontrada em outros tecidos, acumulado nestes ceratócitos anormais. Yassa et al., em $1987^{(11)}$, descreveram a presença de depósitos de imunoglobulina no terço posterior do estroma corneano, tanto intracelular como extracelular em um paciente com distrofia filiforme.

Holopainen et al., em $2003^{(12)}$, descreveram os achados da microscopia confocal em um paciente com distrofia pré-Descemet. Observaram a presença de inclusões de alta reflectividade no estroma corneano imediatamente anterior a membrana de Descemet tanto intracelular como extracelular. A espessura corneana avaliada pela microscopia confocal foi de $656 \mu \mathrm{m}$, sendo que as inclusões foram encontradas no estroma posterior entre $625-645 \mu \mathrm{m}$. A biomicroscopia ultra-sônica se mostrou um exame de pouca utilidade na avaliação desta distrofia, visto que as alterações corneanas eram muito discretas para serem identificadas no exame.

Devido à ausência de relatos de casos em crianças e da possibilidade do material encontrado no interior dos ceratócitos ser semelhante à lipofucsina, alguns autores acreditam que algumas das distrofias préDescemet possam estar relacionadas com processo de envelhecimento da córnea, representando, portanto, um processo degenerativo e não uma distrofia verdadeira. Novos estudos devem ser realizados para que então essas possibilidades possam ser confirmadas.

A distrofia policromática posterior, considerada um tipo de distrofia pré-Descemet, foi primeiramente descrita por Fernandez-Sasso et al., em $1979^{(13)}$. Desde então, nenhum novo relato foi feito referente a esta distrofia na literatura. As principais diferenças entre a distrofia policromática e as outras distrofias préDescemet são: a variedade de cores das opacidades quando em iluminação direta ou indireta da lâmpada de fenda, a distribuição uniforme das opacidades por toda a córnea sem formar áreas de agregação, e os espaços transparentes entre as opacidades maiores que a própria opacidade. Neste mesmo trabalho, ao estudarem 46 membros da mesma família com distrofia policromática posterior puderam deduzir que a doença foi transmitida de forma autossômica dominante, estando presente em quatro gerações sucessivas. No presente trabalho, quando se estudou a família do caso 1 , três membros apresentavam a distrofia, mostrando uma possível transmissão do tipo autossômica dominante.

Atualmente acredita-se que as distrofias préDescemet, como a distrofia policromática, são originadas de uma desordem primariamente genética, provavelmente autossômica dominante.

Este trabalho é o primeiro relato de casos de distrofia policromática posterior na literatura nacional, e o segundo na literatura mundial.

Por ser uma distrofia com alterações corneanas discretas e que não causa redução na acuidade visual de seus portadores, é possível que exista um número considerável de casos não diagnosticados em nosso meio. Esperamos com o presente trabalho estar contribuindo para facilitar a identificação de novos casos da distrofia policromática posterior e, dessa forma, permitir que novos conhecimentos sejam adicionados aos atuais.

\section{ABSTRACT}

The authors describe four rare cases of polychromatic posterior corneal dystrophy, not describe in national literature. The opacities are deep in the stroma, dotlike, polychromatic, uniform in size, distributed from limbus to limbus, leading no reduction in visual acuity. It is also presented a bibliographic review of pre-Descemet's dystrophy.

Keywords: Corneal dystrophies, hereditary; Corne/ pathology; Chromosome disorders; Case reports [Publication type] 


\section{REFERÊNCIAS}

1. Belfort Júnior R, Kara-José N. Córnea - clínica cirúrgica. São Paulo: Roca; 1996. p.339-48.

2. External disease and cornea: Basic and clinical science course Section 8. San Francisco: American Academy of Ophthalmology; 2000/2001. p. 283-311.

3. Vogt A. Cornea farinata. Schweizerische Medizinische Wochenschrift. 1923; 53:991.

4. Maeder G, Danis P. On a new form of corneal dystrophy (deep filiform dystrophy of the cornea) associated with keratoconus. Ophthalmologica. 1947; 114:246-8.

5. Franceschetti A, Chodos J, Dieterle P, Forni S. [Deep filiform dystrophy of the cornea]. Bull Mem Soc Fr Ophtalmol. 1957; 70:175-9. French.

6. Strachan IM, Maclean H. Posterior polymorphous dystrophy of the cornea. Br J Ophthalmol.1968; 52(3):270-2.

7. Franceschetti A, Maeder G. [Deep cornea dystrophy in a case of congenital ichthyosis]. Bull Mem Soc Fr Ophtalmol. 1954; 67:1469; discussion 149-50. French.

8. Collier M. [Heredo-familial nature of predescemetic punctiform dystrophy]. Bull Soc Ophtalmol Fr. 1964; 64:731-4. French.
9. Grayson M, Wilbrandt H. Pre-descemet dystrophy. Am J Ophthalmol. 1967; 64(2):276-82.

10. Curran RE, Kenyon KR, Green WR. Pre-Descemet's membrane corneal dystrophy. Am J Ophthalmol. 1974; 77(5):711-6.

11. Yassa NH, Font RL, Fine BS, Koffler BH. Corneal immunoglobulin deposition in the posterior stroma. A case report including immunohistochemical and ultrastructural observations. Arch Ophthalmol. 1987; 105(1):99-103.

12. Holopainen JM, Moilanen JA, Tervo TM. In vivo confocal microscopy of Fleck dystrophy and pre-Descemet's membrane corneal dystrophy. Cornea. 2003; 22(2):160-3.

13. Fernandez-Sasso D, Acosta JE, Malbran E. Punctiform and polychromatic pre-Descemet's dominant corneal dystrophy. Br J Ophthalmol. 1979; 63(5):336-8.

\section{ENDEREÇO PARA CORRESPONDÊNCIA:}

\section{Patrick Frensel de Moraes Tzelikis}

SQN 203, Bloco G, apt ${ }^{\circ} 405$

CEP 70833-070 - Brasília - DF

E-mail: tzelikis@terra.com.br 